\title{
The importance of "first-blood circulation stage", a new insight into the pathogenesis of clinical manifestations of preeclampsia $^{*}$
}

\author{
Lucijan Mohorovic, Vladimir Micovic
}

Department of Environmental Medicine, University of Rijeka School of Medicine, Rijeka, Croatia

Email: lucijan.mohorovic@pu.t-com.hr

Received 6 August 2012; revised 10 September 2012; accepted 23 October 2012

\begin{abstract}
The tested hypothesis points out that exposure to environmental toxic substances originating from coal or other fossil fuels burning is the most decisive for the impacts of the metabolic synergy of nitrogen oxides as oxidants that cause hemoglobin oxidation to methemoglobin, and sulphur dioxide metabolites as inhibitors of antioxidants, in the bloodstream throughout the period of pregnancy. The main difference between the present three-stage hypothesis and other hypotheses is the assertion that, in the pathogenesis of early and late complicated pregnancy, methemoglobin takes on an important role. Methemoglobin by itself and from heme, redox-active ferric iron as a product of methemoglobin catabolism, has prooxidant properties and causes important structural and functional changes in the vascular endothelium, such as growth arrest, senescence, morphological alterations and cell apoptosis. Our own prospective study of methemoglobin in pregnancy revealed a significant rise and correlation between the ground level of $\mathrm{SO}_{2}$ and the level of methemoglobin: $>1.5 \mathrm{~g} / \mathrm{L}(\mathrm{r}=\mathbf{0 . 7 2}$, $\mathrm{p}$ $<0.01)$ and sulfhemoglobin $(r=0.53, p<0.05)$ in the bloodstream of pregnant women which can be explained on the basis of an oxidant-antioxidant imbalance (nitrogen and sulphur synergy effects), resulting in methemoglobinemia. Methemoglobinemia and pregnancy complications like stillbirths recorded throughout the exposure period are significantly higher than those recorded in the control period $(p=0.0205)$, and the frequencies of reproductive loss were significantly lower in the control than in the exposure period ( $p<$ 0.05). We point out the importance of the "FirstBlood circulation stage" as a new insight into the pathogenesis of the multisystemic oxidative stress and the link between a poorly perfused placenta and clinical manifestations of preeclampsia. The results suggest that the level of methemoglobin concentration
\end{abstract}

\footnotetext{
*The authors declare no conflict of interest.
}

in the bloodstream of pregnant women correlate with the inhalation of substances generated from coal combustion ( $\mathrm{SO}_{2}, \mathrm{NO}_{\mathrm{x}}, \mathrm{NO}, \mathrm{NO}_{2}$ and others) and that is an early biomarker of the identification of women with a pregnancy risk, and having an significant role upon adverse effects on mother and fetus health.

Keywords: Methemoglobinemia; Mother and Fetal Preeclampsia; Three-Stage Disorders; Environmental Oxidants; Biomarker; Nitrogen and Sulphur Synergy

\section{INTRODUCTION}

As a main aim we want to make a contribution to the establishment of sources of oxidants as key factors in understanding the role oxidants play in the pathogenesis of cardiovascular endothelial dysfunction.

Pregnancy anemia is well known, however aquired methemoglobinemia and sulfhemoglobinemia, as added factors of hypoxemia, have still not been sufficiently researched in human reproduction studies. Environmental oxidants have an influence on the level of methemogobinemia and sulfhemoglobinemia, but are, as yet, neglected factors. The Two Stage model of the evolution of preeclampsia now underpins much preeclampsia research, and is based on the perception of a poorly perfused placenta (Stage 1 ) and the production of processes leading to the clinical manifestations of preeclampsia (Stage 2) [1]. The present hypothesis about preeclapsia as a multisystemic disease is explained by the long-term impact of environmental oxidants, based on the role of the synergy between exogenously derived nitrogen oxides $\mathrm{NO}_{\mathrm{x}}(\mathrm{NO}$, $\mathrm{NO}_{2}$ ) and sulphur compounds in the development of oxidative stress on erythrocytes, and change the hemoglobin to methemoglobin which then causes adverse effects on the vascular endothelium. The formation of methemoglobin-ferric iron (Fe III) from hemoglobin-ferrous iron (Fe II) leads to the destruction of erythrocytes, and the free hemoglobin from hemolysis can be directly cyto- 
toxic and alter the state of endothelial cells, promoting hemolysis-associated smooth muscle dystonia, vasculopathy and endothelial dysfunction [2,3].

\section{METHODS AND RESULTS}

To confirm the hypothesis of complications caused by the inhalation of environmental toxins in the population, a subset of pregnant women living around the TE Plomin1 (coal burning plant) in Labin, Croatia, was investigated. Pregnant women living within $3.5-12 \mathrm{~km}$ of the power plant were included in the study. This coalpowered thermoelectric plant is the single major air polluter in the district of Labin (about 25,500 residents), Istria, Croatia. Pregnant women were selected for the research target group from patients of the Primary Health Center, which is responsible for the health of about 25,500 residents, of which about 6180 women of reproductive age had permanently assigned obstetrics care. Subjects were informed about the purpose of the research and gave written consent. At the time of the study, 260 women were considered representative based on the criteria that they were pregnant and came to the center for regular monthly checkups during the clean and dirty periods.

Because of the lack of evidence of fetal methemoglobin, the findings of proper prospective studies of mother methemoglobinemia in human pregnancy have been used [4]. The aim of the prospective study was to determine whether the level of methemoglobin concentration in the bloodstream of pregnant women correlated with the inhalation of substances generated from coal combustion $\left(\mathrm{SO}_{2}, \mathrm{NO}_{\mathrm{x}}, \mathrm{NO}, \mathrm{NO}_{2}\right.$ and others). Since the plant was closed from 19 February 1989 to 6 September 1989, it was possible to produce results from two separate periods: the "clean" period (April 1989 to July 1989) and the “exposure” period (December 121989 to March 1990).
Every hour of operation, the plant emits 8.5 tons (18.080 $\mathrm{mg} / \mathrm{m}^{3}$, or $\left.6900.8 \mathrm{ppm}\right)$ of sulphur dioxide in addition to nitrogen oxides, carbon dioxide, carbon monoxide, TSP (Total Suspended Particles) and other coal combustion products. The coal used for firing has a high sulphur content $(9 \%-11 \%)$ and a very high level of radioactivity (ca. $300 \mathrm{~Bq} / \mathrm{kg}$-i.e. 10 to 15 times higher than the average for other types of coal in the world). In both periods, the "clean period" and the "dirty period", clinically uncomplicated and complicated pregnancies were included. The following parameters were analyzed: hemoglobin (Figure 1), methemoglobin and sulfhemoglobin (Figure 2) and urine sulfate and pH (Figure 3).

The MetHb and SulfHb levels (Ref. values MetHb < $1 \%$, SulfHb $0.0 \%-0.4 \%$ ) in the bloodstream of the pregnant women were determined in blood samples taken three times with a 1-month period between each test in the course of regular monthly obstetric check-ups and analyzed in the laboratory by spectrophotometer based on the difference in absorbance between MetHb and cyanmethemoglobin, which method measures all forms of hemoglobin except sulfhemoglobin. The determination of SHb by spectrophotometry is based on the fact that the molecules $\mathrm{HbO}_{2}$ and $\mathrm{SHb}$ have a maximum absorption at $620 \mathrm{~nm}$. $\mathrm{HbO}_{2}$ will disappear when a solution of KCN is added whereas SHb will still remain in the solution.

For the analysis we used the Chi-square analysis test and statistical linear correlation tests. The research indicated the presence of a significantly positive correlation between the level of methemoglobin $(r=0.72$, $\mathrm{p}<0.01)$, and sulfhemoglobin $(r=0.53, p<0.05)$ in the bloodstream of pregnant women and the daily ground level concentration of $\mathrm{SO}_{2}$ from coal combustion.

Using linear correlation statistical tests, methemoglobinemia and stillbirths, recorded over the "exposure" pe-

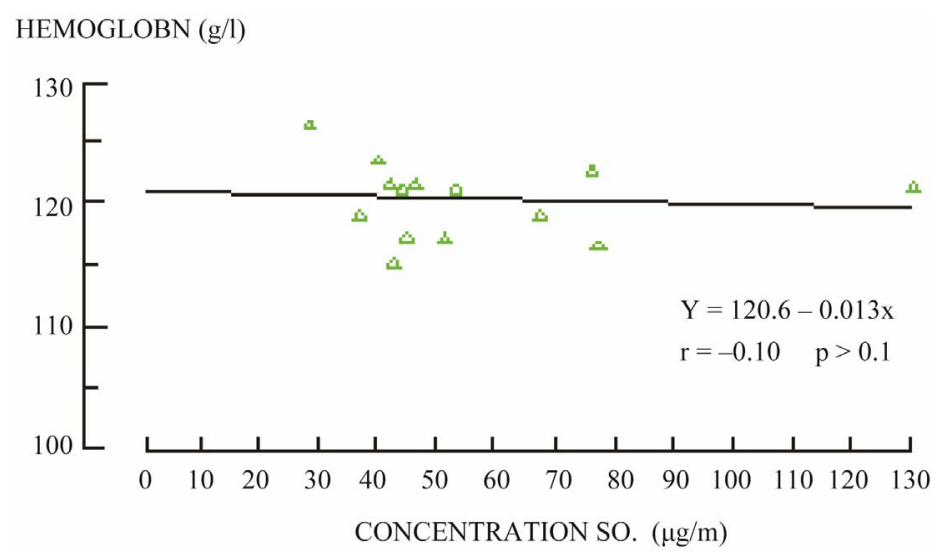

Figure 1. Correlation between ground level of $\mathrm{SO}_{2}$ and average value of hemoglobin in blood of pregnant women in the exposure period. Source: L. Mohorovic: "Influence of coal combustion products on pregnancy course in microregional conditions”. Rigeka, 1991. 


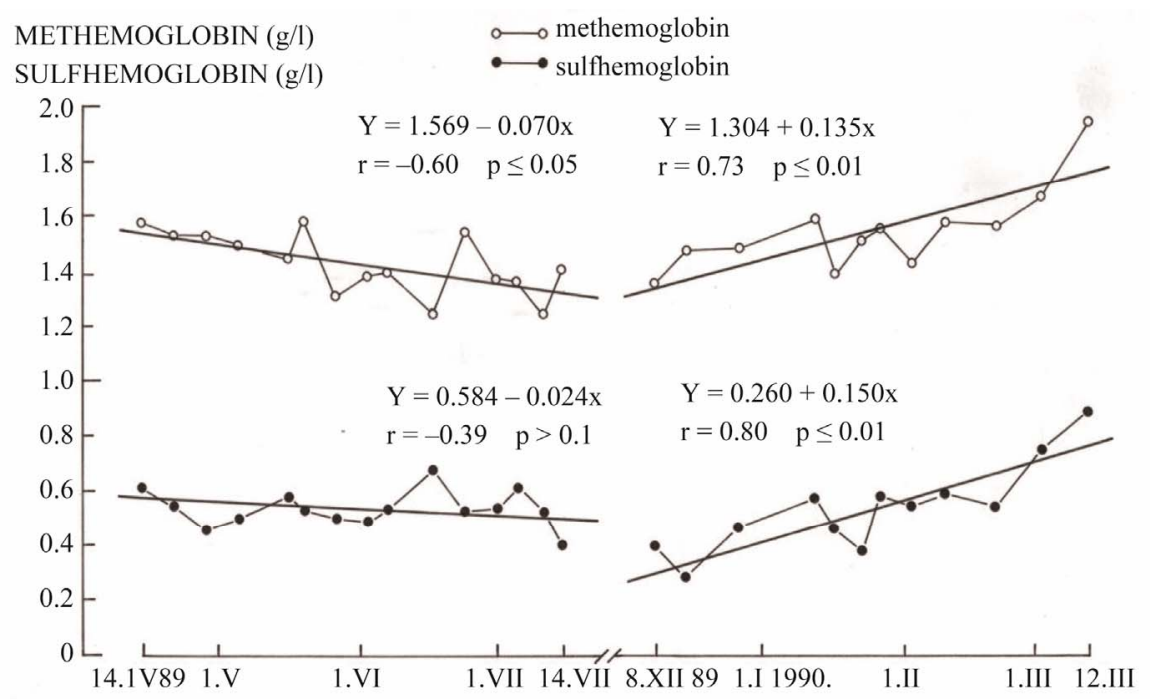

Figure 2. Trend of the level of the methemoglobin and sulfhemoglobin in blood of pregnant women on "clean", when TE Plomin coal power plant was no operational, and the “dirty” period when it was operational (1989-1990).

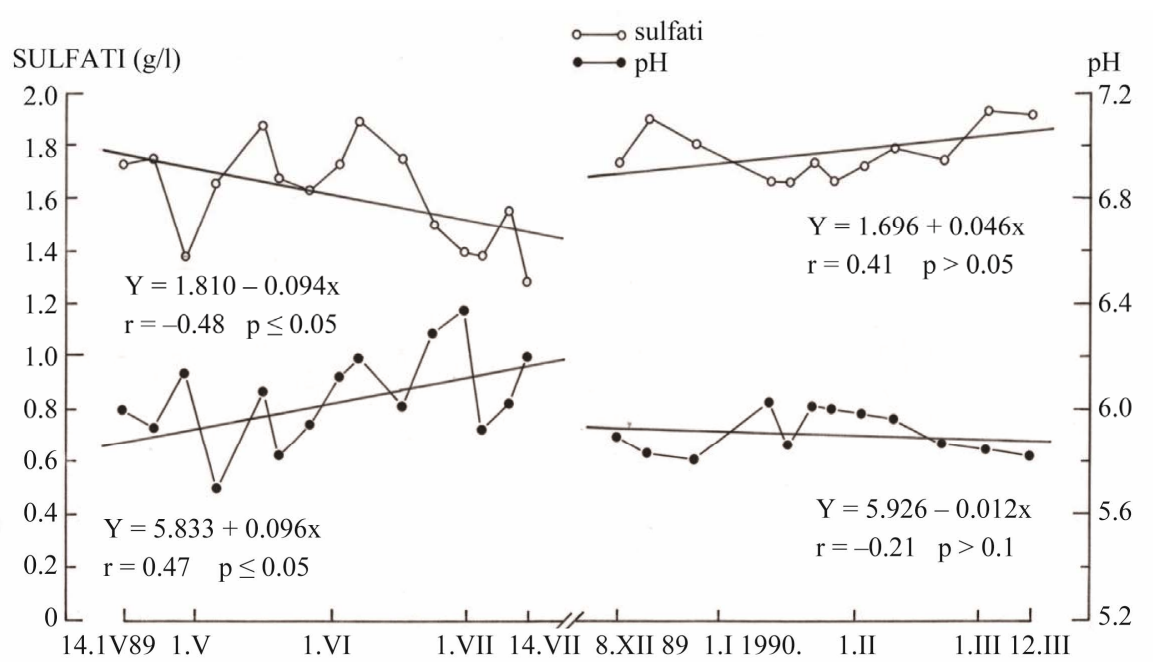

Figure 3. Trend of the avarage value of sulphates and $\mathrm{pH}$ in urine of pregnant women on the "clean", when TE Plomin coal power plant was no operational, and the "dirty" period when it was operational (1989-1990).

riod, were significantly higher than in the "control" pe$\operatorname{riod}(p=0.0205)$ and the frequencies of miscarriages and stillbirths were significantly lower in the "control" period than in the "exposure" period $(\mathrm{p}<0.05)$. In 1987 the coal power plant operated for 4772 hours. In 1988 and 1989, it worked at the lower capacity of 2754 and 579 work hours respectively. In 1987 we found an increased and statistically (Chi-square test) significant number of pre-mature births $(\mathrm{p}=0.026)$ and a relative risk of 1.76 in comparison to 1988 and 1989 (normalized to coal power plant working hours).

A proper retrospective study confirms the role of inhaled environmental toxins in the early development of the human embryo and in adverse pregnancies caused by permanent oxidative stress, the unbalanced production of reactive oxygen species (ROS), reactive nitrogen species (RNS), reactive sulphur species (RSS), and other unfavorable metabolic processes in early embryo genesis, resulting in growth-arrested cells. The results show a statisticaly significant link between the exposure emissions of $\mathrm{SO}_{2}$ in the first two gestational months, and the length of gestation $(-0.0914, p=0.008)$ as well as the body mass of the newborn baby $(-0.0807, \mathrm{p}=0.016$, Pearson correlation). The time of the greatest susceptibility in human gestation is the period of organ formation during weeks 3 - 8 following fertilization. Available data indicate that the consequences of exposure depend on the nature of chemicals, their targets, and the timing of ex- 
posure relative to critical windows in the development of the reproductive system, when the embryo is particularly sensitive to toxic agents and when the effects tend to be irreversible [5].

\subsection{The Significance of Methemoglobinemia in Relation to Environmental Oxidants and as a Precursor of Adverse Effects upon Mother and Fetus Health, Manifested as Three Stage Disorders}

The most important question remains unanswered: whether oxidative (or nitrative) stress is causative of the disorders or a consequence of a disease which may or may not have clinical relevance [6].

The inhalation of continuous but not extremely high levels of nitrogen oxides (nitrogen dioxide and nitric oxide) from the time of preconception, which enter the mother's blood circulation directly through the alveolar-capillary membrane where nitrites and nitrates (indices of nitric oxide production) as oxidants, transform hemoglobin into its pathological reversible form methemoglobin, which alters vascular reactivity. Methemoglobin by itself has prooxidant properties, induces structural and functional changes in the vascular endothelium, and leads to the destruction of erythrocytes and other conesquences [7]. When the blood level of irreversible methemoglobin rises as a result of failed methemoglobin reduction, the patients may present signs of hypoxia, which may lead to coma and ultimately death, owing to the concentration level of methemoglobin from early and/or in late complicated pregnancies.

\subsection{First Stage (Blood Circulation Stage)}

Continuous exposure to strong exogenous oxidants such as $\mathrm{NO}_{\mathrm{x}}\left(\mathrm{NO}\right.$ and $\mathrm{NO}_{2}$ ) reversibly oxidizes oxyhemoglobin $\left(\mathrm{Fe}^{2+}\right)$ to methemoglobin $\left(\mathrm{Fe}^{3+}\right)$ and irreversible methemoglobinemia can arise because of the disruption of the oxidant/antioxidant balance supported by $\mathrm{SO}_{2}$ metabolites, as inhibitors of antioxidants, by the synergistic degradation of antioxidant thiols. Methemoglobin by itself and from heme, redox-active ferric iron as a product of methemoglobin catabolism, has prooxidant properties and causes important structural and functional changes in the vascular endothelium such as growth arrest, senescence, morphological alterations and cell apoptosis. Methemoglobinemia is a condition in which hemoglobin oxidized to the ferric form (Fe III) is unable to transport oxygen to tissue and causes hypoxia. In healthy women MetHb contributes to less than $1 \%$ of the hemoglobin level [8] but its level rises in pathophysiological conditions when red blood cells are affected by genetic, xenobiotic, pharmaceutical, idiopathic or toxic agents from food and chemical compounds from the environment [9].
In pathological conditions, MetHb catabolises into heme, and the activity of heme-oxygenase eventually leads to products such as bilirubinbiliverdin, carbon monoxide, and Ferric iron (Fe III) with paramagnetic and toxic properties. Iron is essential for normal cell function, but it also generates toxic Reactive Oxygen Species (ROS) that adversely affect the vascular endothelium and the blood-brain barrier. These changes include growth arrest, senescence, morphological alterations and cell apoptosis, and they lead to both vessel thrombosis and endothelium denudation under the influence of redoxactive ferric iron [10].

\subsection{Second Stage (Deciduo-Placentation Stage)}

Continued chronic exposure ( $>24 \mathrm{~h}$ a day) to inhaled oxidants prolongs systemic vascular endothelial dysfunction and the spiral arteries of the decidua and myometrium, a reproductive "locus minoris resistentiae" and a susceptible organ, and fail to establish an adequate uteroplacental blood flow. This condition is thought to give rise to relatively hypoxic trophoblast tissue, and may promote an exaggerated state of oxidative stress in the placenta [11]. From the initiation of pregnancy, inhaled oxidants may have adverse effects on placental development and particularly on the sensitive embryo and fetus "target" organs in the critical period of organogenesis to miscarriage, IUGR and preterm birth. Such effects occur as a consequence of local reduced trophoblast invasion, decreased uteroplacental blood perfusion and an increased change of placental activity. The decrease of intensity or changeability of continuity of exposure to oxidants can explain the enigma of compromised fetal growth without preeclampsia mother symptoms.

\subsection{Third Stage (Systemic Disease Stage)}

Preeclampsia is defined as gestational hypertension combined with the onset of maternal renal, hepatic, hematological or neurological dysfunction or of fetal restriction. As a "systemic disease" it can occur in the second half of pregnancy as a consequence of a locally reduced trophoblast invasion and reduced utero-placental blood perfusion. Continuous exposure to inhaled toxic substances in addition to oxidants by food, water, drugs and their associated cumulative effects, causes increased oxidative stress. This is in accordance with the more recently increased attention to the multisystemic nature of the syndrome linked with the involvement of almost all organs [12,13]. Consistent findings provided evidence that in the end-stage of the disease, when the continuous action of oxidants exhausts the mother's own self-defense (enzyme and non-enzyme antioxidants imbalance), the increased production of ROS, superoxide anion and hydrogen peroxide, reduced nitric oxide synthesis, and the decreased bioavailability of antioxidants, and were 
demonstrated to play a pivotal role in experimental and human hypertension [14]. When the blood level of ireversible methemoglobin rises as a result of failed methemoglobin reduction, patients may present signs of hypoxia, which may lead to coma and ultimately death, in the event of a $70 \%$ rise in the concentration level of methemoglobin. Symptoms of increased methemoglobin in the mother are presented as visual disturbances, headache, dyspnea, pallor, cyanosis, palpitations, chest pain, confusion and delirium up to tonic-clonic convulsions, coma and death.

The author personally observed that these symptoms were also common in severe anemia, preeclampsia and eclampsia, suggesting that methemoglobinemia may also be a precursor for these latter conditions. When a high level of methemoglobin becomes irreversible, the deficiency of antioxidants persists and oxidative stress continues, attacking the vascular endothelium of the kidneys, the brain and other vital organs and the tissues of the mother. This can result in eclampsia and/or death as the final form of "maternal preeclampsia". As the oxidants possess the capacity to cross the damaged fetomaternal placental barrier, “fetal preeclampsia” becomes manifest [9]. Under these adverse conditions, levels of methemoglobin in the fetus rise, also resulting in preterm birth, stillbirth or early neonatal death. We use the term "fetal preeclampsia” because, along with excessive maternal exposure to environmental oxidants, the fetus, in its more susceptible pathophysiological phase, is also involved. At this stage, laboratory measurements of blood lipid peroxide and glutathione levels as markers of oxidative stress, and the methemoglobin concentration in maternal blood as a biomarker of individual exposure, can reliably predict an adverse pregnancy, which may end in stillbirth or early neonatal death.

\section{DISCUSSION}

The tested hypothesis points out that exposure to environmental toxic substances originating from coal or other fossil fuels burning is the most decisive for the impacts of the metabolic synergy of nitrogen oxides as oxidants that cause hemoglobin oxidation to methemoglobin, and sulphur dioxide metabolites as inhibitors of antioxidants, throughout the period of pregnancy.

A proper hypothesis originally presents the connection of oxidative stress process in three stages manifesting as alterations on the mother, the placenta and the fetus, and so explains away the enigma about the connection between Stage 1, the failed vascular remodeling of the vessels that supply the placental bed, and Stage 2, the multisystemic maternal syndroma of preeclampsia. As an assertion of this hypothesis Tabacova et al. confirmed that maternal methemoglobin was strongly associated with cord methemoglobin ( $\mathrm{p}<0.0001)$. Mean maternal methemoglobin values were approximately twofold higher in the mothers of infants born prematurely and in fetal distress than in mothers who bore normal babies. According to these authors, high levels of lipid peroxides were strongly associated with preterm birth and with exposure to oxidized nitrogen compounds (RNS) [15,16]. There have been numerous reports on adverse reactions to sulphate agents describing reactions of ingested, inhaled and parentaly administered sulphate [17]. There is mounting evidence that "reactive sulphur species" (RSS) with stressor properties are formed under conditions of oxidative stress, as the second-generation rapidly oxidizes and subsequently inhibits the functions of thioldependent proteins and enzymes. Rust et al. found that severe preeclampsia had higher plasma concentrations of endothelin-1 compared to normotensive patients, but the origin of this increase remains uncertain [18]. In our opinion it occurs because the oxidative stress process starts in the upper respiratory airway as a result of endothelial dysfunction and causes the build up of endothelin-1 in the mother's circulation. Schiff et al. consider that high levels of endothelin-1 in the fetoplacental circulation may be pathophysiologically important in fetal growth retardation [19]. In line with this, Hjelt et al. measured the level of newborn fetal methemoglobin. Thirty-three cases (8\%) from 415 neonates were methemoglobin positive (MetHb 6\%), with mean methemoglobin being 19\% (range $6.5 \%-45.5 \%$ ) and about $40 \%$ of neonates born at 25 - 30 weeks of gestation and $60 \%$ with a birth weight $<$ 1000 g were methemoglobin positive [20].

\section{CONCLUSION}

We particularly emphasize the importance of the "FirstBlood circulation stage" as a new insight into the pathogenesis of the multisystemic oxidative stress and the link between a poorly perfused placenta and clinical manifestations of preeclampsia, and contribute to resolve the dilema about whether oxidative (or nitrative) stress is causative of the disorders or a consequence of a disease which may or may not have clinical relevance. The results suggest that methemoglobin as an individual indicator of oxidative stress is an early biomarker of the identification of women with a pregnancy risk. Methemoglobinemia as a disorder from the impacts of the metabolic synergy of nitrogen oxides as oxidants and sulphur dioxide metabolites as inhibitors of antioxidants, have a significant role as a precursor of the adverse effects on mother and fetus health, clinicaly manifested as "mother preeclampsia” and "fetal preeclampsia".

\section{REFERENCES}

[1] Roberts, J.M. and Hubel, C.A. (2009) The two stege model of preeclampsia: Variations on the theme. Placenta, 


\section{0, S32-S37.}

[2] Jeney, V., Balla, V., Yachie, A., Arga, Z., Vercellotti, G.M., Eaton, J.W. and Balla, G. (2002) Pro-oxidant citotoxic effects of circulatiing heme. Blood, 100, 879-887.

[3] Woollard, K.J., Sturgeon, S., Chin-Dusting, J.P., Salem, H.H. and Jackson, S.P. (2009) Erythrocyte hemolysis and hemoglobin oxidation promote ferric chloride-induced vascular injury. The Journal of Biological Chemistry, 284, 13110-13118.

[4] Mohorovic, L. (2003) The level of maternal methemoglobin during pregnancy in air polluted environment. Environmental Health Perspectives, 11, 1902-1905. doi:10.1289/ehp.6055

[5] Mohorovic, L. (2004) First two months of pregnancycritical time for preterm delivery and low birth weight caused by the adverse effects of coal combustion toxins. Early Human Development, 80, 115-123.

[6] Poston, L., Chappell, L., Seed, P. and Shennan, A. (2011) Biomarkers of oxidative stress in pre-eclampsia. Pregnancy Hypertension: An International Journal of Womem's Cardiovascular Health, 1, 22-27. doi:10.1016/j.preghy.2010.10.009

[7] Starodubtseva, M.N., Ignatenko, V.A. and Cherenkevich, S.N. (1999) Damage to erythrocytes caused by the interaction of nitrite-ions with hemoglobin. Biofizika, 44, 1068-1072.

[8] Kinoshita, A., Nakayama, Y., Kitayama, T., Tomita, T. and Tomita, M. (2007) Simulation study of methemoglobin reduction in erythrocytes. Differential contributions of two pathways to tolerance to oxidative stress. FEBS Journal, 274, 1449-1458.

[9] Mohorovic, L. (2007) The role of methemoglobinemia in early and late complicated pregnancy. Medical Hypotheses, 68, 1114-1119. doi:10.1016/j.mehy.2006.09.053

[10] Jaffe, E.R. (1981) Methemoglobin pathophysiology. Progress in Clinical Biological Research, 51, 133-151.

[11] Myatt, L. (2002) Role of placenta in preeclampsia. Endocrine, 19, 103-111. doi:10.1385/ENDO:19:1:103

[12] Huppertz, B. (2008) Placental origins of preeclampsia:
Challenging the current hypothesis. Hypertension, 51, 970-975.

[13] Sankaralingam, S., Arenas, I.A., Lalu, M.M. and Davidge, S.T. (2006) Preeclampsia: Current understanding of the molecular basis of vascular dysfunction. Expert Reviews in Molecular Medicine, 8, 1-20.

[14] Touyz, R.M. (2004) Reactive oxygen species, vascular oxidative stress, and redox signaling in hypetension: What is the clinical significance? Hypertension, 44, 248252. doi:10.1161/01.HYP.0000138070.47616.9d

[15] Tabacova, S., Balabaeva, L. and Little, R.E. (1997) Maternal exposure to exogenous nitrogen compounds and complications of pregnancy. Archives of Environmental Health, 52, 341-347.

[16] Tabacova, S., Baird, D.D. and Balabaeva, L. (1998) Exposure to oxidazed nitrogen: Lipid peroxidation and neonatal health risk. Archives of Environmental Health, 53, 214-221. doi:10.1080/00039899809605698

[17] Etlik, O., Tomur, A., Tuncer, M., Ridvanagaol, A.Y. and Andac, O. (1997) Protective effects of antioxidant vitamins on red blood cell lipoperoxidation induced by $\mathrm{SO}_{2}$ inhalation. Journal of Basic \& Clinical Physiology \& Pharmacology, 8, 31-43. doi:10.1515/JBCPP.1997.8.1-2.31

[18] Rust, O.A., Bofill, J.A., Zappe, D.H., Hall, J.E., Burnett, J.C. and Martin, J.N. (1997) The origin of endothelin1 in patients with severe preeclampsia. Obstetrics \& Gynecology, 89, 754-757. doi:10.1016/S0029-7844(97)00093-8

[19] Schiff, E., Weiner, E., Zalel, Y., Mashiach, S., Sihai, B.M. and Shalev, E. (1994) Endothelin1, 2 levels in umbilical vein serum of intra-uterine growth retarded fetuses as detected by cordocentesis. Acta Obstetricia et Gynecologica Scandinavica, 73, 21-24. doi:10.3109/00016349409013387

[20] Hjelt, K., Lund, J.T., Sherling, B., Bendixen, S., Lundstrom, K., Stovring, S., Voldsgaard, P. and Linnet, K. (1995) Methemoglobinemia among neonates in an neonatal intensive care unit. Acta Paediatrica, 84, 365-370. doi:10.1111/j.1651-2227.1995.tb13650.x 